\title{
Research on Electrochemical Properties of Flexible Graphite - Activated Carbon Supercapacitor
}

\author{
Quanmao Yü \\ Institute of Functional Materials of Jiangxi University of Finance \& Economics, Nanchang 330013, \\ P.R. China;
}

Keywords: Flexible Graphite; Activated carbon; Supercapacitor; Electrochemical properties

\begin{abstract}
Flexible graphite - activated carbon based tetraethylammonium tetrafluoroborate propylene carbonate solution supercapacitor was synthesized. The electrochemical properties of supercapacitor (1.0 M tetraethylammonium tetrafluoroborate propylene carbonate solution) with different amount of flexible graphite were studied. The electrochemical performance of supercapacitor with $5.0 \%, 8.0 \%, 10.0 \%$ and $15.0 \%$ of flexible graphite replace the activated carbon in composite electrode indicate that the $5 \%$ flexible graphite supercapacitors have the largest specific capacitance $112.89 \mathrm{~F} / \mathrm{g}$, the resistance is the least $3.5 \Omega$. The electrochemical performance of supercapacitor with $5.0 \%, 8.0 \%, 10.0 \%$ and $15.0 \%$ of flexible graphite replace the carbon black in composite electrode show that the $10.0 \%$ flexible graphite supercapacitors have the largest specific capacitance $122.79 \mathrm{~F} / \mathrm{g}$, but the $8.0 \%$ carbon black replaced supercapacitors have the smallest resistance, which is least $4.3 \Omega$.
\end{abstract}

\section{Introduction}

Supercapacitors offer a promising alternative approach to meeting the increasing power demands of energy-storage system in general and of portable (digital) electronic devices due to their excellent electrochemical performance [1-4]. Materials used in supercapacitors electrode must have large surface area, relatively good electrical properties and moderate cost [4]. Activated carbon (AC) is the mostly used electrode materials. Flexible graphite (also named expandable graphite) is a kind of new carbon material, have properties of extremely strong resistance to high and low temperature, good conductivity and large specific surface area, is likely to have great potential be applied in super capacitor as electrode materials [5-8].This work, flexible graphite - activated carbon based tetraethylammonium tetrafluoroborate propylene carbonate solution supercapacitor was synthesized. The electrochemical properties of supercapacitor $(1.0 \mathrm{M}$ tetraethylammonium tetrafluoroborate propylene carbonate solution) with different amount of flexible graphite were studied.

\section{Experiment}

\section{Reagents and materials}

AC, tetraethylammonium tetrafluoroborate / propylene carbonate solution (1.0 mol L-1, AR grade), flexible graphite, polytetrafluoroethylene emulsion (AR grade), conductive carbon black, conductive adhesive and battery separator were all commercial products and purchased from market. All these chemicals and materials were employed as purchased without any further purification unless specified.

\section{Materials preparation and synthesis of supercapacitor}

The AC and flexible graphite were soaked in $2.0 \mathrm{M}$ sodium hydroxide solution and ultrasonic (KQ-50DE) dispersed 5 hrs (power: $85 \%$ ). Then infrared dried $6 \mathrm{hrs}$ in air at $140{ }^{\circ} \mathrm{C}$ after washed by water till the $\mathrm{pH}$ reaching 8.0. Then infrared dried for $12 \mathrm{hrs}$ in air at $110{ }^{\circ} \mathrm{C}$. The treated AC, conductive carbon black and polytetrafluoroethylene emulsion were mixed in an agate mortar with 
the wt. ratio of 70: 25: 5 (two groups sample: a. 5.0\%, 8.0\%, 10.0\% and 15.0\% of flexible graphite replace the activated carbon, b. $5.0 \%, 8.0 \%, 10.0 \%$ and $15.0 \%$ of flexible graphite replace the carbon black ). Then the mixture was rolled to thin slices with the thickness of $0.65 \mathrm{~mm}$ and punched to diameter $2.8 \mathrm{~cm}$ disc electrode layers, and then vacuum infrared dried $12 \mathrm{hrs}$ at $110{ }^{\circ} \mathrm{C}$. The electrode layers were sticked to steel discs by conductive adhesive forming supercapacitor electrode and soaked in Et4NBF4 PC solution for 30 minutes. Two supercapacitor electrodes were separated by battery separator and assembled to a supercapacitor.

\section{Capacitance characterization}

The capacitance studies on the capacitors were made on LAND battery tester (CT2001A) and impedance measurement unit, IM6ex ZAHNER, Messsysteme (Kroanch, Germany) at room temperature. The capacitance values, the capacitance values - charge and discharge cycle number relationship, cyclic voltammetry curve were obtained.

\section{Results and Discussions}

\section{Electrochemical Properties of Flexible Graphite Replace Activated Carbon Supercapacitor}

Fig. 1 shows the specific capacitance of supercapacitors with different amounts flexible graphite replaced active carbon. It can be seen from the Fig. 1 that 5.0\% replaced supercapacitors have the largest specific capacitance, reach $112.89 \mathrm{~F} / \mathrm{g}$. Even largeer than the supercapacitors with whole active carbon (that is $104.13 \mathrm{~F} / \mathrm{g}$ ).

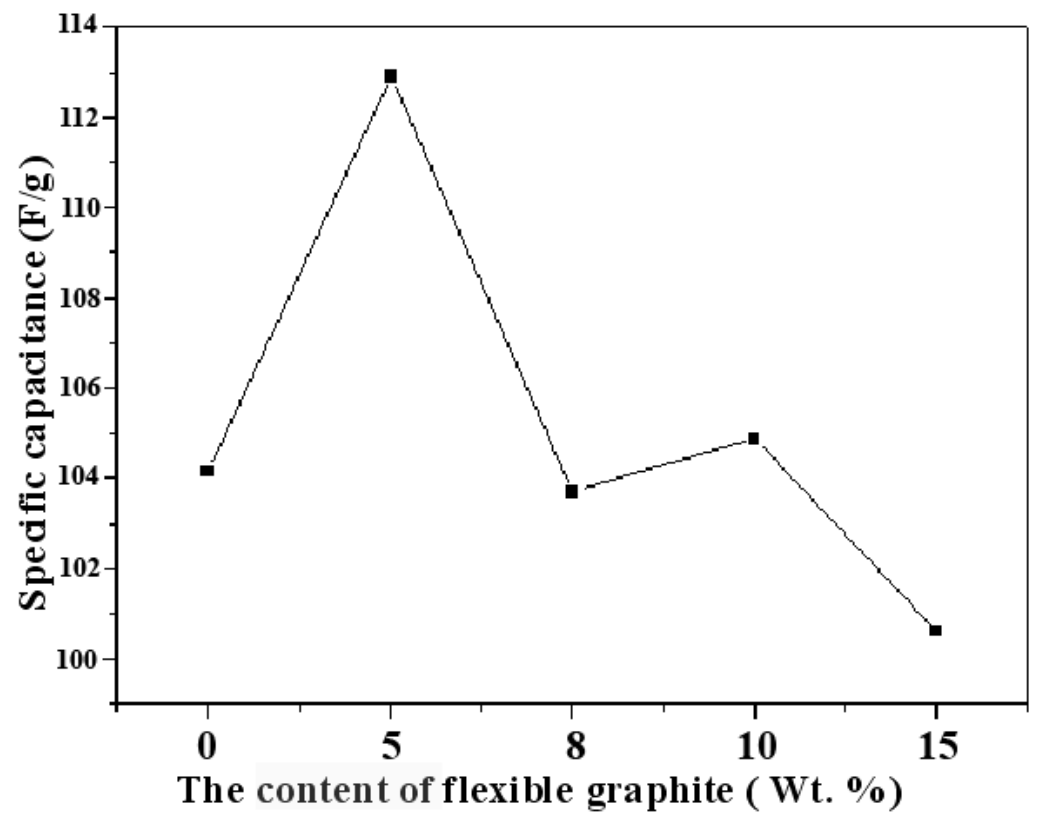

Fig.1 The specific capacitance of supercapacitors with different amounts flexible graphite replaced active carbon

Fig. 2 shows the resistance of supercapacitors with different amounts flexible graphite replaced active carbon. It can be seen from the Fig. 2 that 5.0\% replaced supercapacitors have the smallest resistance, reach $3.5 \Omega$. Much lower than the supercapacitors with whole active carbon (that is about $6.0 \Omega)$. 


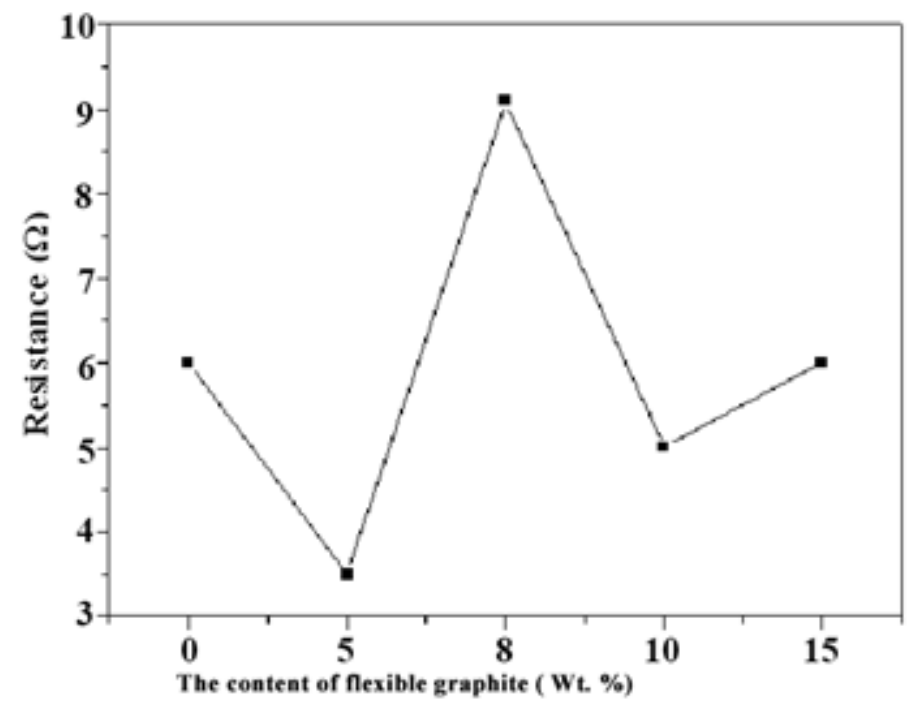

Fig. 2 The resistance of supercapacitors with different amounts flexible graphite replaced active carbon

\section{The Electrochemical Properties of Flexible Graphite Replace Carbon Black Supercapacitor}

Fig. 3 shows the specific capacitance of supercapacitors with different amounts flexible graphite replaced carbon black. It can be seen from the Fig. 3 that 10.0\% replaced supercapacitors have the largest specific capacitance, reach $122.79 \mathrm{~F} / \mathrm{g}$. Even larger than the supercapacitors with whole active carbon ( that is $104.13 \mathrm{~F} / \mathrm{g}$ ).

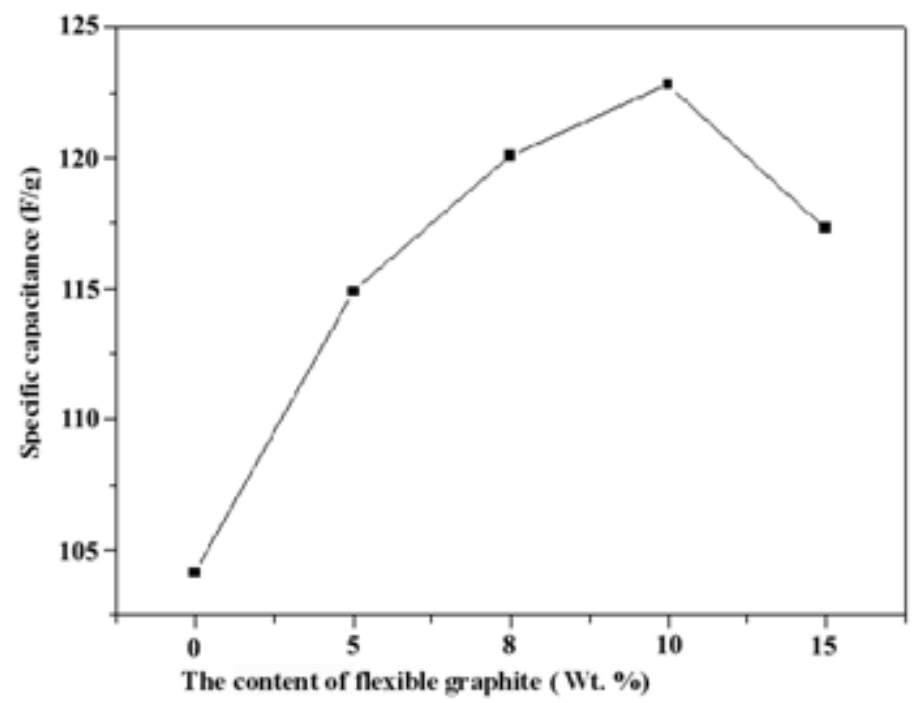

Fig.3 The specific capacitance of supercapacitors with different amounts flexible graphite replaced carbon black

Fig. 4 shows the resistance of supercapacitors with different amounts flexible graphite replaced carbon black. It can be seen from the Fig. 4 that 8.0\% replaced supercapacitors have the smallest resistance, reach $4.3 \Omega$. Much lower than the supercapacitors with whole active carbon (that is about $6.0 \Omega)$. 


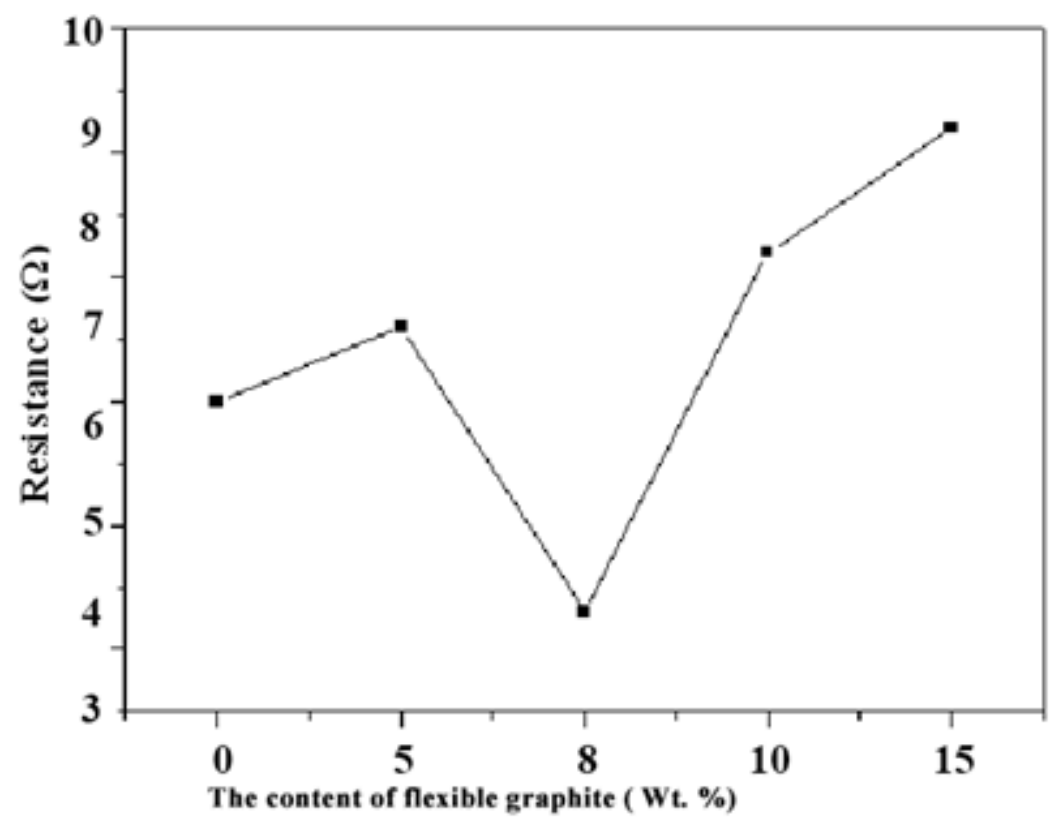

Fig. 4 The resistance of supercapacitors with different amounts flexible graphite replaced carbon black

\section{Conclusions}

Flexible graphite - activated carbon based tetraethylammonium tetrafluoroborate propylene carbonate solution supercapacitors have following characteristics:

The supercapacitors with $5 \%$ activated carbon replaced by flexible graphite have the largest specific capacitance $112.89 \mathrm{~F} / \mathrm{g}$, the resistance is the least $3.5 \Omega$.

The supercapacitors with $10.0 \%$ carbon black replaced by flexible graphite have the largest specific capacitance $122.79 \mathrm{~F} / \mathrm{g}$, but the $8.0 \%$ carbon black replaced supercapacitors have the smallest resistance, which is least $4.3 \Omega$.

\section{Acknowledgements}

This work was financially supported by the National Natural Science Founds of China (61204003), the Jiangxi Natural Science Foundation (20133BAB20005,), Jiangxi Provincial Department of Education (GJJ12270), the Floor Plan of Science and Technology for Higher School of Jiangxi Province ( KJLD13034), and the Excellent Young Academic Talent Program of Jiangxi University of Finance and Economics, respectively.

\section{References}

[1] Meigen Deng, Yingfang Chen, Yuanyuan Li: Electronic Components and Materials (In Chin), Vol. 27(2008), p. 1

[2]Volker Presser, Min Heon, Yury Gogotsi: Adv. Funct. Mater. Vol. 21 (2011), p. 810

[3] A.G. Pandolfo, A.F. Hollenkamp: J. of Power Sources Vol. 157 (2006), p. 11

[4] Lili Zhang, X. S. Zhao: J. Chem. Soc. Rev. Vol. 38(2009), p. 2520

[5] Chunyu Guo, Chengyang Wang, Jingyuan Chen: Chinese Journal of Power Sources (In Chin) Vol. 30(2006), p. 929 
[6] Limei Bo, Boying Liu: Chem. Eng. (In Chin) Vol. 23(2009), p.14

[7] D.D.L. Chung: J. Mater. Eng. Perform. Vol. 9(2000), p.161

[8] Liu Qinfu, D. A. Spears, Qinpu Liu: Appl. Clay. Sci. Vol. 18(2001), p. 89 